\title{
Cabazitaxel for Metastatic Castration-Resistant Prostate Cancer: Retrospective Data Analysis from an Indian Centre
}

\author{
Vanita Noronha Amit Joshi Vamshi Krishna Muddu Vijay Maruti Patil \\ Kumar Prabhash \\ Department of Medical Oncology, Tata Memorial Hospital, Mumbai, India
}

\section{Keywords}

Cabazitaxel · Prostate-specific antigen · Disease-free survival · Neutropenia - Leucopenia

\begin{abstract}
Objective: To determine the efficacy and safety of cabazitaxel in metastatic castrationresistant prostate cancer (mCRPC) patients from the named patient programme (NPP) at our centre. Methods: $\mathrm{mCRPC}$ patients who progressed on docetaxel were given cabazitaxel intravenously every 3 weeks until disease progression or unacceptable toxicity occurred. Overall survival, progression-free survival, prostate-specific antigen response, quality of life (QOL) changes, and safety were reported. Results: Nine men received cabazitaxel (median: 7 cycles; range: 1-27) under the NPP and were followed until death. Median survival was 14.07 months (1.07-23.80) and progression-free survival was 2.67 months (1.07-20.27). QOL was stable for most patients. Common adverse events (grade $\geq 3$ ) were neutropenia $(n=8)$, anaemia $(n=4)$, and leucopenia $(n=4)$. Conclusion: These data from 9 patients are consistent with the results reported in the TROPIC study with a manageable safety profile.
\end{abstract}

(C) 2016 The Author(s)

Published by S. Karger AG, Basel 


\section{Case Reports in Oncology}

\section{Introduction}

Global estimates indicate that prostate cancer is the second most commonly diagnosed cancer and the sixth leading cause of cancer mortality among men [1]. As per this report, the age-standardised incidence rate for prostate cancer in Indian men was 3.7 per 100,000 persons during the year 2008, which is much lower than the estimates from developed countries [1]. However, the number of new prostate cancer patients in India is expected to rise, not only due to the increase in population size and average life expectancy contributing to absolute numbers, but also due to increasing incidence rates attributed to changing lifestyles $[2,3]$.

Trend analyses of five population-based cancer registries (Mumbai, Chennai, Bangalore, Delhi, and Bhopal) using data collected over a period of 20 years (1982-2003) reveal that the incidence of prostate cancer was on the rise across all registries in India [2]. Another study that analysed time trends of prostate cancer for the period 2005-2008 using data from various registries shows that the age-adjusted incidence rates vary widely across India from 0.8 to 10.9 per 100,000 person-years, which is attributed to differences in diet and lifestyle practices between different regions [3]. The highest age-adjusted incidence rates were seen in urban centres such as Delhi (10.9), Bangalore (8.9), Mumbai (7.5), and Pune (7.5). Overall, factors such as rapid urbanisation and changing lifestyles, increased awareness, changes in diagnostic methods, prostate-specific antigen (PSA) screening, and better access to medical care will further result in a rising burden of prostate cancer in India [3-5].

For patients with advanced or metastatic prostate cancer, androgen ablation is the standard first-line therapy, but eventually most of them develop progressive disease. Subsequent to the development of metastatic castration-resistant prostate cancer (mCRPC), treatment options are very limited. Docetaxel is the first-line chemotherapy for mCRPC. For mCRPC patients who experience disease progression during or after docetaxel treatment, there were no therapeutic alternatives that improved survival until the recent US Food and Drug Administration (FDA) approval of cabazitaxel. This approval was given based on the data from the phase III TROPIC trial, in which MCRPC patients who had progressed on previous treatment with docetaxel demonstrated a significant improvement in survival and clinical benefit with cabazitaxel plus prednisone compared to those treated with mitoxantrone plus prednisone [6].

Cabazitaxel is a new-generation tubulin-binding taxane selected from among more than 400 compounds due to its activity in taxane-resistant cell lines [7]. A named patient programme (NPP) was initiated in India by Sanofi from April 2011 until its commercial availability in India.

The objective of this paper is to report the efficacy and safety data from 9 patients who were treated with cabazitaxel in a single centre (Tata Memorial Hospital, Mumbai, India) under the NPP.

\section{Subjects and Methods}

\section{Design}

We collected data on the patients in the cabazitaxel NPP recorded as a part of routine clinical practice, independent of Sanofi's NPP. We retrospectively analysed the medical records of these patients for efficacy, quality of life (QOL) changes, and the safety of cabazitaxel. 


\section{Case Reports in Oncology}

Noronha et al.: Cabazitaxel for Metastatic Castration-Resistant Prostate Cancer: Retrospective Data Analysis from an Indian Centre

\section{Patients}

The cabazitaxel NPP included patients above 18 years of age with mCRPC that had progressed during or after completion of docetaxel therapy, had undergone surgical or medical castration, had an Eastern Cooperative Oncology Group (ECOG) performance status of 0-2, had a life expectancy $\geq 3$ months, had adequate bone marrow, hepatic, and renal function, had no history of severe hypersensitivity to cabazitaxel, prednisone, or drugs formulated with polysorbate 80 , had no active grade $\geq 2$ peripheral neuropathy or stomatitis, and had no other serious illness (including secondary cancer).

\section{Procedures}

Cabazitaxel was intravenously administered at a dose of $25 \mathrm{mg} / \mathrm{m}^{2}$ over $1 \mathrm{~h}$ on day 1 of every 21-day cycle. In addition, all patients received therapy with oral prednisone at $10 \mathrm{mg}$ daily. Before initiating the cabazitaxel infusions, the patients received appropriate premedication with antihistaminic, corticosteroid (dexamethasone $8 \mathrm{mg}$ or equivalent), $\mathrm{H}_{2}$ receptor blocker, and antiemetic medications as required. Prophylactic and therapeutic use of granulocyte colony-stimulating factor was followed as per the American Society of Clinical Oncology (ASCO) recommendations [8].

Baseline evaluations included medical history, ECOG performance status, physical examination, serum PSA evaluation, and relevant laboratory tests. Other investigations were performed as per clinical requirements for MCRPC and included CT scanning as well as abdomen and pelvis and bone ultrasound scanning to establish the extent of metastatic disease. Before each cabazitaxel cycle and at the end of treatment, a physical examination as well as relevant blood tests including complete blood counts, liver and renal function tests, and PSA were repeated. Complete blood counts were additionally performed on days 1 and 8 of each treatment cycle and repeated if clinically required. QOL assessments were conducted as per institutional practice at baseline and at each visit using the European Organisation for Research and Treatment of Cancer (EORTC) Core QOL Questionnaire (EORTC QLQ-C30) and the EORTC QOL Questionnaire-Prostate Module (EORTC QLQ-PR25) [9, 10].

The patients were continued on treatment until disease progression or unacceptable toxicity. Toxicity management was followed as described in the prescribing information document on cabazitaxel. In case of severe chemotherapy toxicity (grade $\geq 3$ neutropenia, diarrhoea, and febrile neutropenia), the subsequent chemotherapy cycle was delayed until the condition improved, and the dose of the subsequent cycles of cabazitaxel was reduced to $20 \mathrm{mg} / \mathrm{m}^{2}$. The patients had the opportunity to discontinue cabazitaxel chemotherapy anytime. The treating physician could decide to stop the therapy anytime based on the patient's clinical condition. All patients were followed up until death.

Overall survival (OS) was evaluated as the time interval from the date of initiation of the first cabazitaxel cycle to the date of death from any cause. Progression-free survival (PFS) was a composite endpoint defined as the time interval from the date of initiation of the first cabazitaxel chemotherapy cycle to progression as indicated by PSA progression, objective evidence of tumour progression, symptomatic deterioration, or death.

PSA response was defined as the proportion of patients achieving a decrease in PSA levels of $\geq 50 \%$ from baseline. The objective response rate was evaluated by Response Evaluation Criteria in Solid Tumors (RECIST) in patients with measurable disease.

Safety was assessed by monitoring for adverse events including abnormal findings in vital signs and physical examination and abnormalities in laboratory tests. All adverse events were graded using Common Terminology Criteria for Adverse Events (CTCAE) version 4.0. 


\section{Case Reports in Oncology}

\section{Statistical Analysis}

The statistical analyses were performed by using SPSS ${ }^{\circledR}$ version 20 . The data were listed and summarised descriptively. Continuous variables were summarised with medians, minimum values, and maximum values. Categorical variables were summarised by numbers and percentages of subjects in each corresponding category.

The analyses were performed on the intention-to-treat population (all patients who were assigned to cabazitaxel treatment). The Kaplan-Meier method was used for survival analysis (OS and PFS). The proportion of patients who demonstrated PSA responses at designated time points are presented. Objective tumour response was evaluated using RECIST version 1.1 in subjects with measurable disease. The overall tumour response rate was defined as the sum of complete and partial responses. The QOL responses were scored and analysed as described by the QOL manuals $[10,11]$. Changes in the QOL score from baseline after treatment were analysed using the Wilcoxon signed-rank test.

The safety analyses included all patients who received at least one dose of cabazitaxel. The type and frequency of adverse events are presented as haematological and nonhaematological using CTCAE terms and grades (version 4.0).

\section{Results}

Overall, there were 9 patients treated with cabazitaxel under the NPP whose data were included in this analysis. The baseline characteristics of the patients were as summarised in table 1 . Bone metastasis was seen in 7 patients $(78 \%)$, and 2 patients $(22 \%)$ had visceral metastasis. Measurable disease was seen in 5 patients (56\%) at baseline. The median PSA level at baseline was $194.25 \mu \mathrm{g} / \mathrm{l}$, and all patients had PSA values above $20 \mu \mathrm{g} / \mathrm{l}$. The median number of cycles of docetaxel received by the patients was 6 , with most patients $(n=7$; $78 \%$ ) having received 4-6 cycles. The median time from the last docetaxel treatment to the first cabazitaxel treatment was 6.83 months (range: 1.4-37.3).

The total number of cabazitaxel cycles delivered to the 9 patients in the NPP was 97. The median number of cabazitaxel cycles delivered was 7 (range: 1-27). There were 3 patients (33\%) who received more than 10 cycles, of whom $2(22 \%)$ received more than 20 cycles. Approximately 69\% (67/97) of the cycles were covered with growth factors. The most common reason $(\mathrm{n}=4 ; 44 \%)$ for cabazitaxel treatment discontinuation was disease progression (online suppl. table 1; for all online suppl. material, see www. karger.com/doi/10.1159/000447710).

All patients were followed up until their death, with the last death recorded in April 2014. The causes of death included disease progression in 8 patients (89\%) and an adverse event (febrile neutropenia) in 1 (11\%). Median OS was 14.07 months (range: 1.07-23.80) (fig. 1).

Disease progression was noted in all 9 patients. This included PSA progression in 5 patients, objective disease progression in 2 patients, and symptomatic progression in 2 patients. Median PFS was 2.67 months (range: 1.07-20.27) (fig. 2).

Of the 9 patients treated with cabazitaxel, there were 8 PSA-evaluable patients who had one baseline and at least one post-baseline PSA measurement. Of these 8 patients, 4 achieved a maximal PSA reduction of $\geq 50 \%$ from baseline after the initiation of cabazitaxel therapy (PSA response rate of 50\%). When the time point of approximately 3 months $(\sim 12$ weeks) after initiation of cabazitaxel therapy was used for assessment of the PSA response, there were 7 PSA-evaluable patients. Of these 7 patients, 2 had a PSA reduction of $\geq 50 \%$ 
from baseline at approximately 12 weeks (PSA response of $29 \%$ at $\sim 12$ weeks) (online suppl. fig. 1). There were 2 patients who demonstrated an objective tumour response (1 complete response and 1 partial response) with cabazitaxel therapy out of the 5 patients with measurable disease at baseline.

The QOL scores (EORTC QLQ-C30 and EORTC QLQ-PR25) at baseline and the best scores attained during cabazitaxel therapy are shown in supplementary table 2 . A trend was noted for maintenance or improvements in EORTC QLQ-C30 functional scales and maintenance or reduction in symptom scales. Similarly, on the EORTC QLQ-PR25 scale, a trend was noted towards improvement in symptom scales. Post-treatment best response was statistically significant for global health status $(\mathrm{p}=0.015)$ and the constipation symptom $(\mathrm{p}=0.017)$ (online suppl. table 2). All patients answered that they had no sexual activity at baseline and after chemotherapy, and none answered the detailed questions on sexual delight; therefore it was not possible to make a reliable analysis of the quality of sexual life. Also some of the patients did not answer the QOL questionnaires during some of the cycles.

The most common adverse events noted with cabazitaxel in this NPP are summarised in table 2 . All patients experienced at least 1 grade $\geq 3$ toxicity. The most frequent haematological grade $\geq 3$ adverse events were neutropenia $(n=8 ; 89 \%)$, anaemia $(n=4 ; 44 \%)$, and leucopenia $(n=4 ; 44 \%)$. Febrile neutropenia was reported in 3 patients $(33 \%)$. The most common non-haematological grade $\geq 3$ adverse events were fatigue $(n=2 ; 22 \%)$, arthralgia $(n=$ $2 ; 22 \%)$, and peripheral sensory neuropathy $(n=2 ; 22 \%)$. Overall there were 4 cases $(44 \%)$ of peripheral neuropathy of all CTCAE grades (peripheral sensory neuropathy: $n=3$; peripheral motor neuropathy: $n=1$ ) and 2 cases $(22 \%)$ of grade 3 peripheral sensory neuropathy. Peripheral neuropathy was seen after 15-17 cycles in these patients. There was 1 death due to an adverse event (the cause of death was febrile neutropenia).

\section{Discussion}

The TROPIC study that resulted in marketing authorisation of cabazitaxel was a phase III, open-label, randomised, multicentre, and multinational trial which demonstrated that cabazitaxel plus prednisone $(\mathrm{n}=378)$ significantly improved OS compared to mitoxantrone plus prednisone $(\mathrm{n}=377)$ in $\mathrm{mCRPC}$ patients who had progressed after docetaxel-based chemotherapy [6]. Our NPP followed eligibility criteria similar to those of the TROPIC study in selecting patients. The baseline profiles of the subjects in this NPP are described in table 1. Many of these patients had poor prognostic disease, with about 5 (56\%) having measurable disease and $2(22 \%)$ with visceral metastases.

The TROPIC study had predefined a treatment duration of a maximum of 10 cycles to decrease the risk of cardiotoxicity that could emerge with mitoxantrone in the comparator arm, while at the same time permitting similar exposures and schedules in the two study arms. The NPP allowed us to continue cabazitaxel dosing until disease progression or unacceptable toxicity. We administered more than 10 cycles of cabazitaxel to 3 patients (33\%), and $2(22 \%)$ of them received more than 20 cycles. These patients showed good tolerance and a favourable response to therapy that allowed its prolonged administration.

In the TROPIC study, the median OS in the cabazitaxel group was 15.1 months, with a median PFS of 2.67 months. In this NPP, a median OS of 14.07 months was seen, with a median PFS of 2.67 months. Though a similar trend in survival outcomes was noticed, it was not conclusive, due to the small sample size and retrospective nature of the study. 
The results of the analysis of QOL scores suggest that QOL was stable during the therapy. Though some of the patients did not answer the questionnaire during some of the cycles, a trend towards improvement in QOL was observed with increasing numbers of cycles. This is similar to results from other studies internationally.

The cabazitaxel expanded access programme in the UK reported a trend towards improved QOL in mCRPC patients treated with cabazitaxel [12]. The Canadian expanded access programme reported statistically significant improvements in general and prostate-specific QOL parameters with cabazitaxel therapy. These researchers also reported improvements in pain scale scores despite the fact that the analgesic doses were stable [13].

Haematological adverse events were frequently seen, and the most common grade $\geq 3$ adverse events were neutropenia, leucopenia, and anaemia (table 2), which is consistent with the safety profile of cabazitaxel reported in clinical studies. The most frequent nonhaematological grade $\geq 3$ adverse events were fatigue $(n=2 ; 22 \%)$, arthralgia $(n=2 ; 22 \%)$, and peripheral neuropathy. When considering adverse events of all CTCAE grades, musculoskeletal pain-related symptoms (pain in extremity: $n=5,55 \%$; arthralgia: $n=4,44 \%$; bone pain: $n=4,44 \%$; back pain: $n=3,33 \%$ ) composed a significant proportion of the events in our subjects. There were 4 cases $(44 \%)$ of all-grade peripheral neuropathy, of which 2 $(22 \%)$ were grade $\geq 3$. Peripheral neuropathy generally occurred after $15-17$ cycles of cabazitaxel in these patients.

There were frequent haematological adverse events, and therefore usage of prophylactic growth factors and monitoring of blood counts need to be considered to prevent or manage them. In addition, a dose reduction to $20 \mathrm{mg} / \mathrm{m}^{2}$ may be considered if patients experience severe toxicities.

\section{Conclusion}

Further studies with cabazitaxel in mCRPC patients, published in India, may help to understand the post-marketing experience better. In the current scenario, mCRPC patients have very few therapeutic options in the post-docetaxel setting in India, and cabazitaxel can be a useful treatment option.

\section{Acknowledgements}

We would like to acknowledge Sanofi India Limited for funding manuscript writing and providing editorial support. We would like to thank Dr. Ashok Krishna and Dr. Rakesh Lalchandani for coordinating with us on behalf of Sanofi India Limited.

\section{Statement of Ethics}

As per the institutional policies, ethics committee approval was not required for earlyaccess programmes like the cabazitaxel NPP. Signed informed consent was obtained for chemotherapy before every cycle of cabazitaxel. 
Noronha et al.: Cabazitaxel for Metastatic Castration-Resistant Prostate Cancer: Retrospective Data Analysis from an Indian Centre

\section{Disclosure Statement}

Sanofi India Limited provided funding for manuscript writing and editorial support.

\section{References}

1 Ferlay J, Shin HR, Bray F, Forman D, Mathers C, Parkin DM: GLOBOCAN 2008 v2.0, Cancer Incidence and Mortality Worldwide: IARC Cancer Base No 10. 2010. International Agency for Research on Cancer. http://globocan.iarc.fr (accessed 15 November 2013).

2 Yeole BB: Trends in the prostate cancer incidence in India. Asian Pac J Cancer Prev 2008;9:141-144.

- 3 Lalitha K, Suman G, Pruthvish S, Mathew A, Murthy NS: Estimation of time trends of incidence of prostate cancer - an Indian scenario. Asian Pac J Cancer Prev 2012;13:6245-6250.

$\checkmark 4$ Hsing AW, Tsao L, Devesa SS: International trends and patterns of prostate cancer incidence and mortality. Int J Cancer 2000;85:60-67.

5 Quinn M, Babb P: Patterns and trends in prostate cancer incidence, survival, prevalence and mortality. Part I: international comparisons. BJU Int 2002;90:162-173.

6 de Bono JS, Oudard S, Ozguroglu M, Hansen S, Machiels JP, Kocak I, et al: Prednisone plus cabazitaxel or mitoxantrone for metastatic castration-resistant prostate cancer progressing after docetaxel treatment: a randomised open-label trial. Lancet 2010;376:1147-1154.

7 Paller CJ, Antonarakis ES: Cabazitaxel: a novel second-line treatment for metastatic castration-resistant prostate cancer. Drug Des Devel Ther 2011;5:117-124.

-8 Smith TJ, Khatcheressian J, Lyman GH, Ozer H, Armitage JO, Balducci L, et al: 2006 update of recommendations for the use of white blood cell growth factors: an evidence-based clinical practice guideline. J Clin Oncol 2006;24:3187-3205.

-9 Aaronson NK, Ahmedzai S, Bergman B, Bullinger M, Cull A, Duez NJ, et al: The European Organisation for Research and Treatment of Cancer QLQ-C30: a quality-of-life instrument for use in international clinical trials in oncology. J Natl Cancer Inst 1993;85:365-376.

10 van Andel G, Bottomley A, Fosså SD, Efficace F, Coens C, Guerif S, et al: An international field study of the EORTC QLQ-PR25: a questionnaire for assessing the health-related quality of life of patients with prostate cancer. Eur J Cancer 2008;44:2418-2424.

11 Fayers PM, Aaronson NK, Bjordal K, Groenvold M, Curran D, Bottomley A: The EORTC QLQ-C30 Scoring Manual. Brussels, European Organisation for Research and Treatment of Cancer, 2001.

12 Bahl A, Masson S, Malik ZI, Birtle AJ, Sundar S, Jones RJ, et al: Cabazitaxel for metastatic castrationresistant prostate cancer (mCRPC): final quality-of-life (QOL) results with safety data from the United Kingdom (UK) Early Access Programme (EAP). J Clin Oncol 2013;31(suppl 6):abstr 91.

13 Winquist E, Sridhar SS, Hubay S, Assi H, Berry SR, Alloul K, et al: Quality of life (QoL) of patients with metastatic castration resistant prostate cancer (mCRPC) treated with cabazitaxel. J Clin Oncol 2013;31(suppl); abstr e16088. 


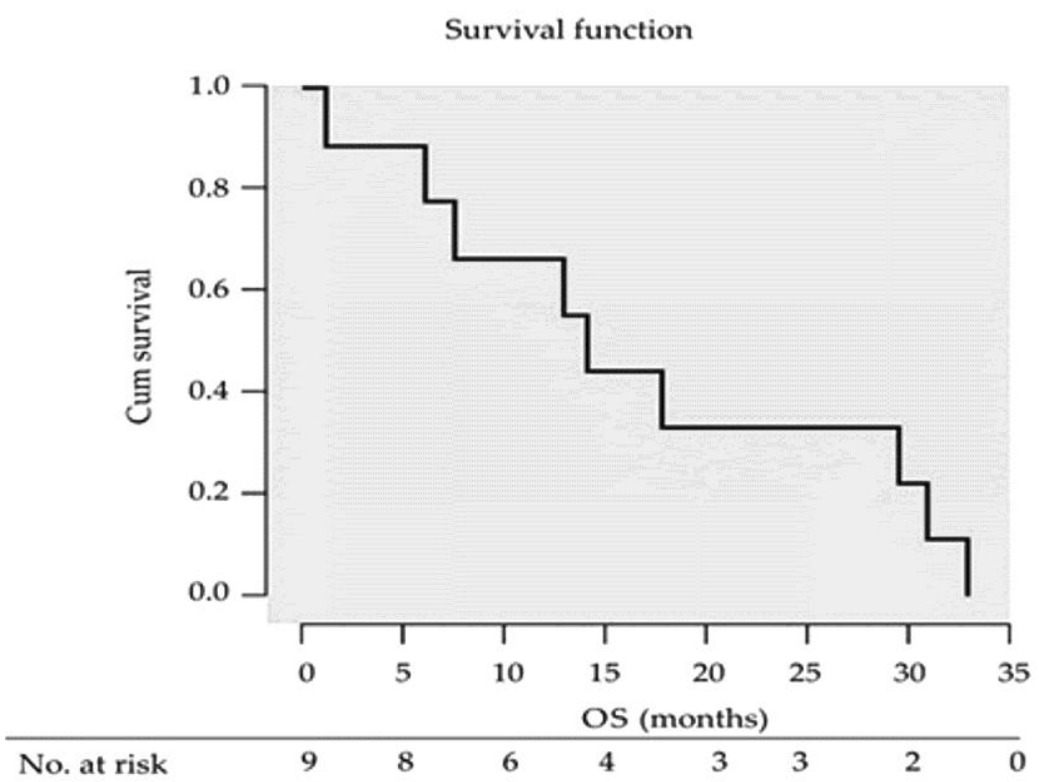

Fig. 1. Overall survival.

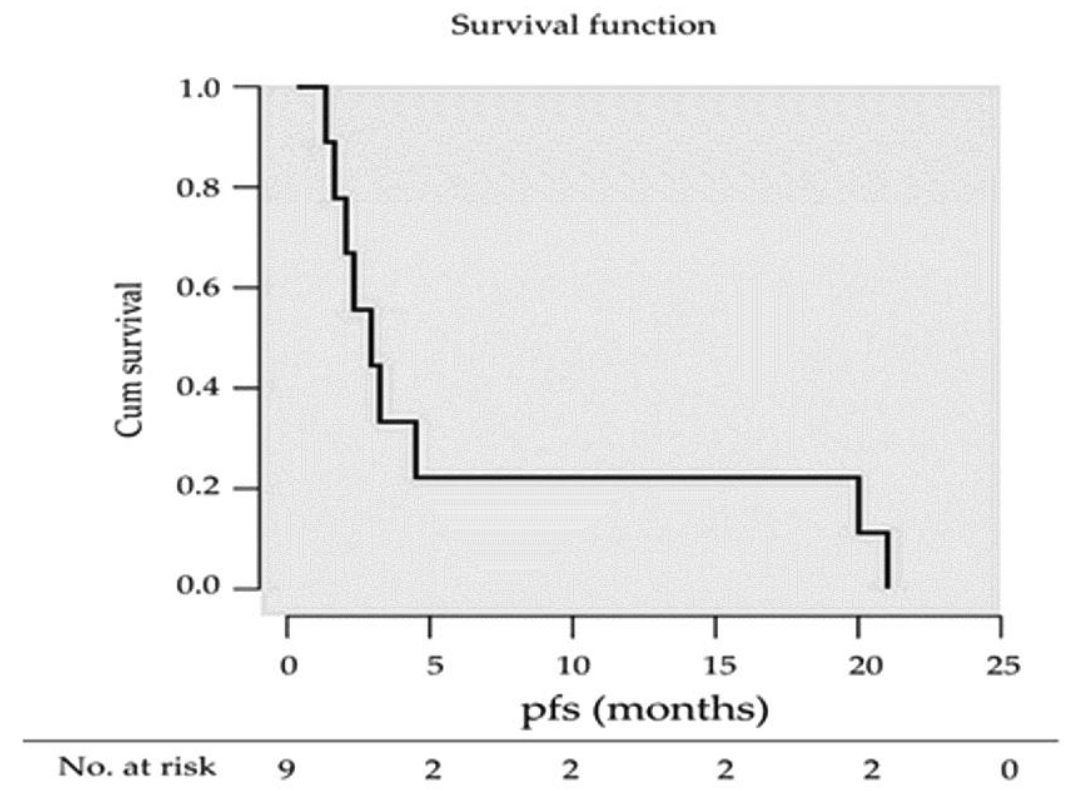

Fig. 2. Progression-free survival. 
Table 1. Baseline characteristics $(n=9)$

\begin{tabular}{|c|c|}
\hline Median age (min., max.), years & $67(54,72)$ \\
\hline Median body mass index (min., max.) & $23.46(18.07,32.52)$ \\
\hline \multicolumn{2}{|l|}{ ECOG performance status } \\
\hline 0 & $3(33)$ \\
\hline 1 & $4(44)$ \\
\hline 2 & $2(22)$ \\
\hline Median & 1 \\
\hline Metastatic disease & $9(100)$ \\
\hline Bone metastases & $7(78)$ \\
\hline Visceral metastases & $2(22)$ \\
\hline Median number (range) of metastatic sites & $2(1-3)$ \\
\hline Median PSA at baseline (min., max.), $\mu \mathrm{g} / \mathrm{l}$ & $194.25(27.26,4,229)$ \\
\hline $\mathrm{PSA}>20 \mu \mathrm{g} / \mathrm{l}$ & $9(100)$ \\
\hline Measurable disease & $5(56)$ \\
\hline \multicolumn{2}{|l|}{ Previous therapy } \\
\hline Hormonal treatment & $9(100)$ \\
\hline Orchiectomy & $8(89)$ \\
\hline LHRH agonists & $2(22)$ \\
\hline Synthetic oestrogens (fosfestrol tetrasodium) & $5(56)$ \\
\hline \multicolumn{2}{|l|}{ Anti-androgens } \\
\hline Bicalutamide & 7 (78) \\
\hline Ketoconazole & $4(44)$ \\
\hline Unspecified anti-androgen & $1(11)$ \\
\hline Previous chemotherapy & $9(100)$ \\
\hline Docetaxel & $9(100)$ \\
\hline Prednisone/prednisolone & $5(56)$ \\
\hline Median number of docetaxel cycles (min., max.) & $6(2,10)$ \\
\hline Docetaxel 1-3 cycles & $1(11)$ \\
\hline Docetaxel 4-6 cycles & 7 (78) \\
\hline Docetaxel $>6$ cycles & $1(11)$ \\
\hline \multicolumn{2}{|l|}{ Median time (range), months from last } \\
\hline docetaxel to first cabazitaxel dose & $6.83(1.4-37.3)$ \\
\hline Previous radiation & 7 (78) \\
\hline Previous biological agents & 0 \\
\hline
\end{tabular}

All values indicate $\mathrm{n}(\%)$ unless specified otherwise. $\mathrm{LHRH}=$ Luteinizing hormone-releasing hormone. 
Noronha et al.: Cabazitaxel for Metastatic Castration-Resistant Prostate Cancer: Retrospective Data Analysis from an Indian Centre

Table 2. Adverse events

\begin{tabular}{|c|c|c|c|}
\hline \multicolumn{2}{|l|}{ CTCAE all grades } & \multicolumn{2}{|l|}{ CTCAE grade $\geq 3$} \\
\hline $\mathrm{AE}$ & n $(\%)$ & $\mathrm{AE}$ & $\mathrm{n}(\%)$ \\
\hline Haematological & & Haematological & \\
\hline Neutrophil count decreased & $8(89)$ & Neutrophil count decreased & $8(89)$ \\
\hline Febrile neutropenia & $3(33)$ & Febrile neutropenia & $3(33)$ \\
\hline Anaemia & $7(78)$ & White blood cell count decreased & $4(44)$ \\
\hline White blood cell count decreased & $5(56)$ & Anaemia & $4(44)$ \\
\hline Platelet count decreased & $4(44)$ & & \\
\hline Lymphocyte count decreased & $3(33)$ & & \\
\hline Non-haematological & & Non-haematological & \\
\hline Fatigue & $7(78)$ & Fatigue & $2(22)$ \\
\hline Anorexia & $7(78)$ & Arthralgia & $2(22)$ \\
\hline Diarrhoea & $7(78)$ & Peripheral sensory neuropathy & $2(22)$ \\
\hline Vomiting & $5(56)$ & Diarrhoea & $1(11)$ \\
\hline Pain in extremity & $5(56)$ & Sepsis & $1(11)$ \\
\hline Arthralgia & $4(44)$ & Blood bilirubin increased & $1(11)$ \\
\hline Bone pain & $4(44)$ & Alkaline phosphatase increased & $1(11)$ \\
\hline Alopecia & $4(44)$ & Bone pain & $1(11)$ \\
\hline Constipation & $4(44)$ & Back pain & $1(11)$ \\
\hline Peripheral neuropathy & $4(44)$ & Pain in extremity & $1(11)$ \\
\hline Peripheral sensory neuropathy & 3 & Muscle weakness in lower limb & $1(11)$ \\
\hline Peripheral motor neuropathy & 1 & Headache & $1(11)$ \\
\hline Back pain & $3(33)$ & & \\
\hline Dysgeusia & $3(33)$ & & \\
\hline Paraesthesia & $3(33)$ & & \\
\hline Oedema on limbs & $3(33)$ & & \\
\hline Nausea & $3(33)$ & & \\
\hline Abdominal pain & $3(33)$ & & \\
\hline
\end{tabular}

$\mathrm{AE}=$ Adverse event. 